\title{
A NOTE ON A LEMMA OF SHELAH CONCERNING STATIONARY SETS
}

\author{
ALAN H. MEKLER, DONALD H. PELLETIER ${ }^{1}$ AND ALAN D. TAYLOR
}

\begin{abstract}
Let $\boldsymbol{\kappa}$ be an infinite cardinal, let $I$ be a nonprincipal ideal on $\kappa$ and let $I^{+}=\{X \subseteq \kappa: X \notin I\} . S(I)$ is the following property of ideals: for every $A \in I^{+}$ and every pair of functions $f, g$ from $A$ into $\kappa$ such that, for every $\alpha \in A$, $f(a) \neq g(\alpha)$, there exists a set $B \subseteq A$ with $B \in I^{+}$such that $f^{\prime \prime} B \cap g^{\prime \prime} B=\varnothing$. We prove that $S(I)$ holds for every weakly selective ideal $I$ on any infinite cardinal $\alpha$ (including $\kappa=\omega$ ), and that $S(I)$ holds for every $\kappa$-complete ideal on $\kappa$ iff $\kappa$ is not strongly inaccessible.
\end{abstract}

Let $\kappa$ be an infinite cardinal. A (proper) ideal on $\kappa$ is a collection $I$ of subsets of $\kappa$ such that $\kappa \notin I$ and whenever $X, Y \in I$ and $Z \subseteq X \cup Y$, then $Z \in I$. If $I$ is an ideal on $\kappa$ then $I^{+}$denotes the sets of "positive $I$-measure"; i.e. $I^{+}=\{X \subseteq \kappa$ : $X \notin I\} . S(I)$ is the following property of ideals: for every $A \in I^{+}$and every pair of functions $f, g$ from $A$ into $\kappa$ such that, for every $\alpha \in A, f(\alpha) \neq g(\alpha)$, there exists a set $B \subseteq A$ with $B \in I^{+}$such that $f^{\prime \prime} B \cap g^{\prime \prime} B=\varnothing$. Shelah's lemma [EM] is the assertion $S\left(N S_{\kappa}\right)$, where $N S_{\kappa}$ is the ideal of nonstationary subsets of the regular uncountable cardinal $\kappa$. The following result will provide a short proof of a generalization of Shelah's lemma.

THEOREM 1. Let $S^{\prime}(I)$ denote the weaker version of $S(I)$ obtained by considering only functions $f$ and $g$ that are one-to-one. Then $S^{\prime}(I)$ holds for every ideal $I$ on every infinite cardinal $\kappa$ (including $\kappa=\omega$ ).

Proof. Let $G$ be the graph on $A$ obtained by making $\alpha$ adjacent to $\beta$ (where $\alpha<\beta)$ iff $g(\alpha)=f(\beta)$. Then each point $B \in A$ is adjacent to at most one $\alpha<\beta$ (since otherwise we would have $f(\beta)=g\left(\alpha_{1}\right)$ and $f(\beta)=g\left(\alpha_{2}\right)$ contradicting the one-to-oneness of $g$ ). Thus each $\beta \in A$ gives rise to a unique decreasing path of finite length. Without loss of generality, assume that the set $B^{\prime}$ of points $\beta \in A$ having such a path of even length is of positive $I$-measure. Since $B^{\prime}$ is clearly an independent set in the graph $G$ it follows that if we have $\alpha, \beta \in B^{\prime}$ with $\alpha<\beta$ then $g(\alpha) \neq f(\beta)$. Now we simply repeat the procedure (starting with $B^{\prime}$ ) with the roles of $f$ and $g$ reversed. The set $B \subseteq B^{\prime}$ of positive $I$-measure so obtained clearly has the property that $f^{\prime \prime} B \cap g^{\prime \prime} B=\varnothing$ as desired.

REMARK. It is worth noting that we really do not need both $f$ and $g$ to be one-to-one-just the "larger." That is, if we let $A_{g}=\{\alpha \in A: f(\alpha)<g(\alpha)\}$ and

Received by the editors August 28, 1980 and, in revised form, March 27, 1981.

1980 Mathematics Subject Classification. Primary 04A20.

Key words and phrases. Ideal, stationary sets, normal ideal, weakly selective ideal.

${ }^{1}$ Research partially supported by the Natural Sciences and Engineering Research Council of Canada under grant \# A8216. 
$A_{f}=\{\alpha \in A: g(\alpha)<f(\alpha)\}$ then either $A_{f} \in I^{+}$or $A_{g} \in I^{+}$. If, for example, $A_{g} \in I^{+}$then we can redo the second step in the above proof so as to appeal to this fact instead of the one-to-oneness of $f$ as follows. Let $G^{\prime}$ be the graph on $B^{\prime}$ in which $\alpha$ is adjacent to $\beta$ (where $\alpha<\beta$ ) iff $f(\alpha)=g(\beta)$. Then each $\alpha$ is adjacent to at most one $\beta>\alpha$ (since $g$ is one-to-one). Notice also that if $\alpha<\beta$ and $\alpha$ is adjacent to $\beta$ then $g(\alpha)>g(\beta)$; that is, $g(\beta)=f(\alpha)<g(\alpha)$. Hence each $\alpha$ gives rise to a unique increasing path of finite length and so we can proceed exactly as in the first part of the proof of Theorem 1.

Recall that an ideal $I$ on $\kappa$ is said to be normal if every regressive function $f$ (i.e. $f(\alpha)<\alpha$ for $\alpha \neq 0$ ) defined on a set of positive $I$-measure is constant on a set of positive $I$-measure. (Fodor's theorem [F] asserts that $N S_{\kappa}$ is normal if $\kappa$ is a regular uncountable cardinal.) $I$ is said to be weakly selective if every function defined on a set of positive $I$-measure is either constant on a set of positive $I$-measure or one-to-one on a set of positive $I$-measure. Weglorz first observed that every normal ideal $I$ is weakly selective. (In fact, if $I$ is normal, $A \in I^{+}, f: A \rightarrow \kappa$ and $f^{-1}(\{\alpha\}) \in I$ for every $\alpha<\kappa$, then the set $B=A-\left\{\inf \left(f^{-1}(\{\alpha\})\right): \alpha<\kappa\right\}$ is in $I$ as can be seen by considering the regressive function $h: B \rightarrow \kappa$ given by $h(\alpha)=$ $\inf \left(f^{-1}(\{\alpha\})\right)$.) Even on uncountable cardinals there are lots of weakly selective ideals that are not normal (e.g. $\left\{X \subseteq \kappa^{+}:|X|<\kappa^{+}\right\}$; for more see [BTW]). With this much said, an easy consequence of Theorem 1 is the following.

COROLlaRY. $S(I)$ holds for every weakly selective ideal I on any infinite cardinal $\kappa$ (including $\kappa=\omega$ ).

Theorem 1 and its corollary suggest the possibility that perhaps $S(I)$ holds for every ideal $I$. This, however, is easily seen not to be the case. For example, if $D$ is an ultrafilter on $\kappa$ and $I$ is the ideal on $\kappa \times \kappa$ dual to $D \times D$, then the projection functions show that $S(I)$ fails. These considerations also show that if $\kappa$ is a measurable cardinal then there is a $\kappa$-complete ideal $I$ (that is, one closed under unions of size less than $\kappa$ ) for which $S(I)$ fails. On the other hand, one can use Theorem 1 (and the remark following it) to show that if $\kappa$ is an infinite successor cardinal then $S(I)$ holds for every $\kappa$-complete ideal on $\kappa$. Hence. if we momentarily agree to call $\kappa$ good iff $S(I)$ holds for every $\kappa$-complete ideal on $\kappa$, then we have that successor cardinals are good and measurable cardinals are not. Our next result will fill the obvious gap (i.e., it will follow that $\kappa$ is good iff $\kappa$ is not strongly inaccessible).

THEOREM 2. For infinite cardinals $\kappa$ and $\mu$, the following are equivalent:

(i)

$$
\kappa \rightarrow\left(\lfloor)_{<\mu}^{2} ;\right.
$$

i.e., for every $f:[\kappa]^{2} \rightarrow \lambda$ where $\lambda<\mu$, there exists $\alpha, \beta$, $\gamma$ such that $\alpha<\beta<\gamma<\kappa$ and $f(\{\alpha, \beta\})=f(\{\beta, \gamma\})$.

(ii) $S(I)$ fails for some proper nonprincipal $\mu$-complete ideal I on $\kappa$. 
Proof. (i) $\rightarrow$ (ii). Assume that

$$
\kappa \rightarrow(\downarrow)_{<\mu}^{2}
$$

and let $A=\{(\alpha, \beta): \alpha<\beta<\kappa\}$. We will construct a $\kappa$-complete proper nonprincipal ideal $I$ on the set $A$ so that the projection functions $\pi_{1}$ and $\pi_{2}$ show that $S(I)$ fails; this clearly suffices.

Let $\mathcal{S}=\left\{X \subseteq A: \pi_{1}(X) \cap \pi_{2}(X)=0\right\}$ and let $I$ be the $\mu$-complete ideal on $A$ generated by $\mathcal{S}$ (i.e., $Y \in I$ iff $Y \subseteq \cup H$ for some $H \subseteq \mathcal{S}$ with $|H|<\mu$ ). Then $I$ is clearly closed downward (i.e., $Y \subseteq X \in I \Rightarrow Y \in I$ ) and under unions of size less than $\mu$. Moreover, every singleton subset $\{(\alpha, \beta)\}$ of $A$ is in $I$ (since $\alpha \neq \beta$ ). Hence, it remains only to show that $I$ is proper.

Suppose not. Then $A=\bigcup\left\{A_{\xi}: \xi<\lambda\right\}$ for some $\lambda<\mu$ where we have $A_{\xi} \in \mathcal{S}$ for each $\xi<\lambda$. We can assume that the $A_{\xi}$ 's are pairwise disjoint. Define $f$ : $[\kappa]^{2} \rightarrow \lambda$ by $f(\{\alpha, \beta\})=\xi$ iff $\alpha<\beta$ and $(\alpha, \beta) \in A_{\xi}$. Since $\lambda<\mu$ and

$$
\kappa \rightarrow(\coprod)_{<\mu}^{2}
$$

we get some $\xi<\lambda$ and $\alpha<\beta<\gamma$ so that $f(\{\alpha, \beta\})=\xi=f(\{\beta, \gamma\})$. But then $(\alpha, \beta) \in A_{\xi}$ and $(\beta, \gamma) \in A_{\xi}$ so $\beta \in \pi_{1}^{\prime \prime} A_{\xi} \cap \pi_{2}^{\prime \prime} A_{\xi}$. This contradicts the fact that $A_{\xi} \in \mathcal{S}$ and thus shows that $I$ is proper.

(ii) $\rightarrow$ (i). Suppose that $h:[\kappa]^{2} \rightarrow \lambda$ for some $\lambda<\mu$ and $h$ shows that

$$
\kappa \nrightarrow\left(\prod\right)_{<\mu}^{2}
$$

Let $I$ be a proper $\mu$-complete ideal on $\kappa$ and suppose that $f, g: A \rightarrow \kappa$ where $A \in I^{+}$and $f(\alpha) \neq g(\alpha)$ for every $\alpha \in A$. For each $\xi<\lambda$ let $A_{\xi}$ be given by

$$
A_{\xi}=\{\alpha \in A: h(\{f(\alpha), g(\alpha)\})=\xi\} .
$$

Since $I$ is $\mu$-complete, $\lambda<\mu$ and $A \in I^{+}$we get that $A_{\xi} \in I^{+}$for some $\xi<\lambda$. Without loss of generality assume that $B \in I^{+}$where $B=\left\{\alpha \in A_{\xi}: f(\alpha)<g(\alpha)\right\}$. Now, to complete the proof it suffices to show that $f^{\prime \prime} B \cap g^{\prime \prime} B=\varnothing$.

Suppose not, and choose $\alpha, \gamma \in B$ such that $f(\alpha)=g(\gamma)=\beta$. Then $f(\gamma)<g(\gamma)$ $=\beta=f(\alpha)<g(\alpha)$ and so $f(\gamma)<\beta<g(\alpha)$. But $h(\{f(\gamma), \beta\})=h(\{f(\gamma), g(\gamma)\})=\xi$ $=h(\{f(\alpha), g(\alpha)\})=h(\{\beta, g(\alpha)\})$ and so the set $\{f(\gamma), \beta, g(\alpha)\}$ contradicts the fact that $h$ shows

$$
\kappa \nrightarrow(l)_{<\mu}^{2} \cdot \square
$$

COROLLARY. For regular cardinals $\kappa$ and $\mu$, the following are equivalent:

(i) $2^{\lambda}>\kappa$ for some $\lambda<\mu$.

(ii) $S(I)$ holds for every $\mu$-complete proper ideal I on $\kappa$. 
Proof. (i) $\rightarrow$ (ii). Assume that $\lambda<\mu$ and $2^{\lambda}>\kappa$. By the previous theorem it suffices to show that

$$
\kappa \nrightarrow(\downarrow)_{\lambda}^{2} ;
$$

our argument here is only a slight (but necessary) modification of the standard example (due to Erdös and Rado [ER]) showing that $2^{\lambda} \nrightarrow(3)_{\lambda}^{2}$. So let $h: \kappa \rightarrow^{\lambda} 2$ be one-to-one where ${ }^{\lambda} 2$ denotes the set of all functions mapping $\lambda$ to 2 . Define $f$ : $[\kappa]^{2} \rightarrow \lambda \times 2$ as follows. If $\alpha<\beta$ then set $f(\{\alpha, \beta\})=(\gamma, i)$ where

$$
\gamma=\inf \{\xi<\lambda: h(\alpha)(\xi) \neq h(\beta)(\xi)\}
$$

and $h(\alpha)(\gamma)=i$. Now, suppose for contradiction that $\alpha<\beta<\delta$ and $f(\{\alpha, \beta\})=$ $(\gamma, i)=f(\{\beta, \delta\})$. Without loss of generality, assume that $i=0$. Then $h(\alpha)(\gamma)=0$ and $h(\beta)(\gamma)=1$ (since $f(\{\alpha, \beta\})=(\gamma, 0))$. But then since $f(\{\beta, \delta\})=(\gamma, 0)$ we have $h(\beta)(\gamma)=0$; contradiction.

(ii) $\rightarrow$ (i). The Erdös-Rado Theorem [ER] asserts that $\left(2^{\lambda}\right)^{+} \rightarrow\left(\lambda^{+}\right)_{\lambda}^{2}$; it follows trivially from this that if $\kappa>2^{\lambda}$ for every $\lambda<\mu$ then

$$
\kappa \rightarrow(\downarrow)_{<\mu}^{2}
$$

The desired result thus follows from the previous theorem.

REMARK. A consequence of the above is that if $\kappa=\sup \left\{\left(2^{\lambda}\right)^{+}: \lambda<\kappa\right\}$ and $\kappa$ is regular, then $S(I)$ fails for some proper nonprincipal $\mu$-complete uniform ideal $I$ on $\kappa$. (To say that $I$ is uniform means that $\{X \subseteq \kappa:|X|<\kappa\} \subseteq I$.)

COROLlaRY. $S(I)$ holds for every $\kappa$-complete proper nonprincipal ideal I on $\kappa$ iff $\kappa$ is not strongly inaccessible.

We conclude with an easy application of the corollary to Theorem 1. An ultrafilter $\mathcal{Q}$ on $\kappa$ is said to be Ramsey if every function $f: \kappa \rightarrow \kappa$ is either constant or a set in $\mathscr{U}$ or one-to-one on a set in $\mathscr{U}$. If $\mathscr{U}$ is an ultrafilter on $\kappa$ and $A$ is a set then a subset $X$ of $A^{\kappa} / \mathcal{Q}$ is called standard if there is a $B \subseteq A$ such that $X=B^{\kappa} / \mathcal{Q}$. We claim that if $\mathscr{U}$ is a Ramsey ultrafilter on $\kappa$, then any two elements of $A^{\kappa} / \mathcal{Q}$ can be separated by a standard set. That is, if $[f],[g] \in A^{*} / \mathscr{Q}$ and $[f] \neq[g]$, then the corollary to Theorem 1 yields a set $X \in \mathcal{Q}$ so that $f^{\prime \prime} X \cap g^{\prime \prime} X=\varnothing$. But now if $B=f^{\prime \prime} X$, then $[f] \in B^{\kappa} / \mathscr{U}$ and $[g] \notin B^{\kappa} / \mathscr{U}$. This application has consequences for certain problems involving cardinalities of ultrapowers; these will appear elsewhere.

\section{REFERENCES}

[BTW] J. Baumgartner, A. Taylor and S. Wagon, Structural properties of ideals, Dissertationes Math. (to appear).

[EM] P. Eklof and A. Mekler, Infinitary stationary logic and abelian groups, Fund. Math. (to appear).

[ER] P. Erdös and R. Rado, A partition calculus in set theory, Bull. Amer. Math. Soc. 62 (1956), 427-489. 
[F] G. Fodor, Eine Bemerkung zur Theorie der regressiven Funktionen, Acta Sci. Math. (Szeged) 17 (1956), 139-142.

Department of Mathematics, University of Western Ontario, London, Ontario, Canada N6a 5 B9

Department of Mathematics, York University, Downsview, Ontario M3J 1P3, Canada (Current address of D. H. Pelletier)

Department of Mathematics, Union College, Schenectady, New York 12308 (Current address of A. D. Taylor)

Current address (A. H. Mekler): Department of Mathematics, Simon Fraser University, Burnaby, British Columbia, Canada V5A 1 A6 